\title{
Alcohol consumption and incident diabetes: The Atherosclerosis Risk in Communities (ARIC) study
}

\author{
Xintong $\mathrm{He}^{1}$ - Casey M. Rebholz ${ }^{1}$ - Natalie Daya ${ }^{1}$ - Mariana Lazo ${ }^{1,2}$ - Elizabeth Selvin ${ }^{1,2}$
}

Received: 18 December 2018 / Accepted: 15 January 2019 / Published online: 28 February 2019

(C) Springer-Verlag GmbH Germany, part of Springer Nature 2019

\begin{abstract}
Aims/hypothesis The aim of this study was to evaluate the prospective association between baseline and 9 year change in alcohol consumption and long-term risk of diabetes and whether these associations might be modified by sex and/or BMI.

Methods We conducted a prospective analysis of 12,042 Atherosclerosis Risk in Communities (ARIC) study participants without prevalent diabetes (55\% women, $78 \%$ white, mean age 54 years). Alcohol consumption was assessed at visit 1 (1987-1989) and visit 4 (1996-1998). We used Cox models to estimate hazard ratios for diabetes risk by baseline drinking categories and change in alcohol consumption, stratified by sex and obesity status.

Results During a median follow-up of 21 years, there were 3795 incident cases of diabetes. Among women, consuming 8-14 drinks/week was associated with a significantly lower risk of diabetes (HR 0.75, 95\% CI 0.58, 0.96) compared with current drinkers consuming $\leq 1$ drink/week. Among men, consuming $8-14$ drinks/week was associated with a borderline significant lower risk of diabetes (HR $0.84,95 \%$ CI $0.70,1.00$ ) and consuming $>14$ drinks/week was associated with a significantly lower risk of diabetes (HR $0.81,95 \%$ CI $0.67,0.97$ ) ( $p_{\text {interaction }}<0.01$ for sex). For both sexes, among current drinkers, there was a significant decreasing trend in diabetes risk as the alcohol consumption increased. The association was modified by BMI ( $p_{\text {interaction }}=0.042$ for women, $p_{\text {interaction }}<0.001$ for men). In women, the inverse association was only seen among overweight and obese participants. In men, the inverse association was more pronounced among obese participants. On average, drinking status did not change substantially over the 9 year period. For men with alcohol intake $\geq 7$ drinks/week at baseline, decreasing alcohol intake was associated with higher risk of diabetes (HR per daily drink decrease $1.12,95 \%$ CI 1.02, 1.23).

Conclusions/interpretation In this community-based population, there was an inverse association between alcohol consumption and diabetes risk. The amount of the alcohol consumption associated with lower risk was different in women and men, and the association was more pronounced among participants with higher BMI.
\end{abstract}

Keywords Alcohol · Diabetes

\section{Abbreviation \\ ARIC Atherosclerosis Risk in Communities}

Electronic supplementary material The online version of this article (https://doi.org/10.1007/s00125-019-4833-1) contains peer-reviewed but unedited supplementary material, which is available to authorised users.

\section{Elizabeth Selvin}

eselvin@jhu.edu

1 Department of Epidemiology, Johns Hopkins Bloomberg School of Public Health, Welch Center for Prevention, Epidemiology, and Clinical Research, 2024 E Monument Street, 2-600, Baltimore, MD 21287, USA

2 Division of General Internal Medicine, School of Medicine, Johns Hopkins University, Baltimore, MD, USA

\section{Introduction}

The incidence of diabetes has increased substantially over the past two decades [1]. Both genetic factors and environmental factors contribute to the risk of diabetes. As a modifiable behaviour, alcohol consumption has been suggested to be relevant to diabetes risk. There is a large body of literature suggesting a protective effect of moderate alcohol consumption on cardiovascular risk and cardiometabolic risk factors, including diabetes [2-4], but the impact of alcohol on cardiovascular and overall population health remains controversial $[5,6]$. Intervention studies have suggested that alcohol 


\section{Research in context}

\section{What is already known about this subject?}

- Moderate alcohol consumption has been associated with lower risk for diabetes

- Whether the association differs by sex or adiposity status is controversial

- Evidence on the association of changes in alcohol consumption and diabetes risk is limited

\section{What is the key question?}

- What is the prospective association of baseline and 9 year change in alcohol consumption with long-term risk of diabetes, and could these associations be modified by sex and/or BMI?

\section{What are the new findings?}

- In this large, community-based cohort of black and white adults, 8-14 drinks/week for women and more than 8 drinks/week for men were associated with a moderately decreased risk of diabetes. In both sexes, among current drinkers, there was a significant decreasing trend in diabetes risk with increasing alcohol consumption

- The inverse associations of alcohol consumption and diabetes risk were more pronounced among individuals with higher BMI

- The amount of alcohol consumed was relatively stable during the 9 year period

\section{How might this impact on clinical practice in the foreseeable future?}

- The association of alcohol consumption with diabetes may differ by sex and adiposity status, suggesting that the pathways by which alcohol impacts risk are potentially complex. In our study, alcohol was associated with a lower risk of diabetes, but this association was more pronounced in adults who were overweight or obese

consumption might improve insulin sensitivity among women [4]. Some prior observational studies have found a protective association of moderate alcohol consumption on diabetes risk, but this association has differed by sex and adiposity in some reports [7-16]. U- or J-shaped associations between alcohol consumption and risk of future diabetes have been reported [ 7 , 9, 11, 14, 15], with evidence in some cohorts for stronger inverse associations in women [8, 10, 13]. Associations by adiposity status have been inconsistent $[8,16]$. Furthermore, few prior studies have examined changes in alcohol consumption and risk of future diabetes in the general population [17].

The objective of the present study was to examine whether associations of alcohol consumption with diabetes risk might differ by sex or BMI in a community-based population with long-term follow-up for incident diabetes. We also undertook analyses to assess changes in alcohol consumption patterns over time with risk of diabetes.

\section{Methods}

Study population The Atherosclerosis Risk in Communities (ARIC) study is a large, ongoing cohort of predominately black and white middle-aged adults from four US communities. A total of 15,792 participants attended visit 1, which took place from 1987 to 1989 . Subsequent visits took place in
1990-1992 (visit 2), 1993-1995 (visit 3), 1996-1998 (visit 4), and 2011-2013 (visit 5).

For the present study, we excluded the small number of participants with race other than black or white $(n=48)$; blacks from Minneapolis, Minnesota $(n=22)$; and blacks from Washington County, Maryland $(n=33)$. We additionally excluded participants with prevalent diabetes $(n=1863)$, as well as those who were missing any of the following information: diabetes status after visit $1(n=78)$, alcohol consumption $(n=85)$, total cholesterol $(n=189)$, fasting glucose (missing glucose or missing fasting status, $n=306)$, education $(n=19)$, hypertension $(n=60)$, BMI $(n=6)$, waist/hip ratio $(n=6)$, smoking status $(n=6)$, household income $(n=742)$, coronary heart disease $(n=252)$ or physical activity $(n=35)$. Thus, the final population analysed in our study included 12,042 participants, of which 6631 were women and 5411 were men (electronic supplementary material [ESM] Fig. 1). Written informed consent was obtained from all participants, and the study protocols were approved by institutional review boards at all study sites.

Assessment of alcohol intake Information on alcohol consumption and the usual amount of alcohol consumption among current drinkers was obtained during ARIC visit 1 (1987 to 1989) and visit 4 (1996 to 1998). Participants were asked 'Do you presently drink alcoholic beverages?' and 'Have you ever consumed alcoholic beverages?'. Never 
drinkers were defined as participants who answered no to these questions and former drinkers as participants who answered no to the first question and yes to the second. Participants who answered yes to the first question (current drinkers) were further categorised into four groups according to their answers to the following questions: 'How many glasses of wine do you usually have per week (4-ounce glasses)?', 'How many bottles or cans of beer do you usually have per week (12-ounce bottles or cans)?', and 'How many drinks of hard liquor do you usually have per week (1.5-ounce shots)?'. Therefore, our analysis included six categories: never drinkers, former drinkers, current drinkers consuming $\leq 1$ drink/week, current drinkers consuming 2-7 drinks/week, current drinkers consuming 8-14 drinks/week, and current drinkers consuming $>14$ drinks/week. We also evaluated 9 year changes (visit 1 to visit 4 ) in alcohol consumption status with risk of diabetes (after visit 4), where the amount of alcohol consumption for non-drinkers was set to zero.

Assessment of incident diabetes For our analyses of baseline alcohol consumption with future diabetes risk, incident diabetes was defined as a fasting glucose level $\geq 6.99 \mathrm{mmol} / \mathrm{l}$ or a non-fasting glucose level $\geq 11.10 \mathrm{mmol} / \mathrm{l}$ (assessed at any of the four follow-up visits, visits 2-5), current use of diabetes medication or self-report of a physician diagnosis of diabetes (assessed at each follow-up visit and during annual telephone calls to all participants occurring following visit 4), or current use of diabetes medication assessed at visit 5 .

Participants with prevalent diabetes at baseline (based on elevated glucose, self-reported diagnosis, or medication use) were excluded. We followed the participants from their first visit (1987-1989) until they developed diabetes, were lost to follow-up, or administrative censoring at 31 December 2015, whichever came first.

Covariates Demographic covariates included age, sex and race/centre. We adjusted for race/centre as a five-category variable: white participants from Forsyth County, NC; white participants from Minneapolis, MN; white participants from Washington County, MD; black participants from Forsyth County, NC; and black participants from Jackson, MS. Smoking status, education, household income and parental history of diabetes were assessed during the in-person visits using standard questionnaires. Total energy intake was derived from a food frequency questionnaire, including total energy intake from dietary and ethanol consumption, but excluding other energy from alcoholic beverages. Blood pressure was measured three times for each participant at baseline and the mean of the second and the third readings was calculated. Participants with a mean systolic blood pressure $\geq 140 \mathrm{mmHg}$, mean diastolic blood pressure $\geq 90 \mathrm{mmHg}$ or using antihypertensive medication were classified as having hypertension. Participants with any of the following were classified as having coronary heart disease: myocardial infarction defined based on a history of myocardial infarction (self-reported or physicianreported) or using electrocardiography data, self-reported heart or arterial surgery, coronary bypass or angioplasty of coronary artery(ies). Physical activity was assessed using the Baecke questionnaire [18]. BMI $\left(\mathrm{kg} / \mathrm{m}^{2}\right)$ and waist/hip ratio was calculated from measured weight, height, waist circumference and hip circumference assessed during the visit.

Statistical analyses We conducted analyses stratified by sex since the association has been reported to differ in men and women. We used multivariable Cox proportional hazards models to estimate the hazard ratios for the associations between alcohol consumption categories at baseline and risk of incident diabetes. Current drinkers reporting $\leq 1$ drink/week served as the reference group in the analyses. Model 1 included age, race/centre and total energy intake. Model 2 included all variables in Model 1 plus education (less than high school, high school degree or vocational school, at least some college or professional school), household income $(<\$ 25,000$ per year, $\$ 25,000-\$ 49,999$ per year, $\geq \$ 50,000$ per year), parental history of diabetes (yes, no), physical activity (sport index, score 1 to 5), HDL-cholesterol, total cholesterol, baseline fasting glucose, coronary heart disease (yes, no); and timevarying BMI (normal weight, overweight, obese), waist/hip ratio (continuous), hypertension history (yes, no), and smoking status (never smokers, former smokers, current smokers). For the missing data for these four time-varying covariates, we carried forward the last observation. We also tested the trend among current drinkers in the two models for both sexes by modelling the four categories of current drinkers as ordinal variables instead of nominal. The risk of diabetes in participants consuming $\geq 2$ drinks/week was compared with that in those consuming $\leq 1 \mathrm{drink} /$ week, among current drinkers, adjusting for all variables in Model 2.

We assessed BMI as a potential effect modifier on the association between alcohol consumption and risk of diabetes. We first modelled baseline BMI as a continuous variable and included the interaction terms in the regression model to test for effect modification using the likelihood ratio test. Second, we conducted analyses stratified by baseline BMI categories: normal weight, $18.5 \leq \mathrm{BMI}<25 \mathrm{~kg} / \mathrm{m}^{2}$; overweight, $25 \leq \mathrm{BMI}$ $<30 \mathrm{~kg} / \mathrm{m}^{2}$; or obese, BMI $\geq 30 \mathrm{~kg} / \mathrm{m}^{2}$. Participants who were underweight (BMI $<18.5 \mathrm{~kg} / \mathrm{m}^{2}$ ) were excluded from this analysis, which constituted $0.9 \%$ of the study population $(n=114)$.

In addition, we assessed the association between the absolute changes in alcohol consumption (from visit 1 to visit 4) with incident diabetes (after visit 4). We used Cox proportional hazards models with change in alcohol consumption modelled using a linear spline with a knot at no change (absolute difference of 0 drinks/week). This analysis was stratified by sex and baseline alcohol consumption frequency $(<7$ drinks/week or $\geq 7$ drinks/week). 
We conducted two sensitivity analyses. First, to address reverse causation (current drinkers in poorer health may be likely to stop drinking prior to the baseline examination), we conducted our analyses after excluding the 1468 participants with cardiovascular disease or cancer at baseline. Second, we examined the association of alcohol consumption with incident cases of diabetes defined based on self-reported diagnoses of diabetes or diabetes medication use only (ignoring cases of diabetes identified only based on glucose measurements).

Two-sided $p$ values $<0.05$ were considered statistically significant. All the analyses were performed using Stata version 14.0 (StataCorp, College Station, TX, USA).

\section{Results}

Among the 12,042 participants (6631 women, 5411 men) included in the present study, 3795 participants developed diabetes (2059 women, 1736 men) during a median follow-up of 21 years (maximum follow-up of 27 years). In the study population at baseline, compared with participants who consumed $\leq 1$ drink/week, participants who consumed more alcohol were more likely to be men rather than women, had higher daily energy intake, higher HDL-cholesterol and higher glucose and were more likely to be current smokers (all $p$ values for trend $<0.001$ ) (Table 1). Education level and household income were associated with alcohol consumption in a U-shaped pattern. Higher alcohol consumption was associated with higher levels of education and household income, with the exception of the group with the highest alcohol consumption ( $>14$ drinks/week), which had education and income levels more similar to the lightest drinking groups (excluding the heaviest drinking group, $p$ values for trend were 0.007 and $<0.001$ for education and income, respectively). Patterns of baseline characteristics by drinking status were generally similar in men and women, except that in men, excluding the heaviest drinking group, there were no significant differences in education or income ( $p$ values for trend were 0.338 and 0.937 , respectively) (ESM Tables 1 and 2).

Among women, in Model 1, drinking 2-14 drinks/week was associated with a lower risk of diabetes compared with the reference group ( $\leq 1$ drink/week); in Model 2, drinking 8-14 drinks/ week was associated with a lower risk of diabetes as compared with the reference group $(\leq 1$ drink/week) (HR $0.75,95 \%$ CI $0.58,0.96$ ) (Table 2). Among men, there were no significant associations of alcohol consumption categories with diabetes risk in the minimally adjusted model (Model 1: age, race/centre and total energy intake). However, in Model 2, consuming 8-14 drinks/week was associated with a borderline significant lower risk of diabetes (HR $0.84,95 \%$ CI $0.70,1.00$ ) and consuming $>14$ drinks/week was associated with a significantly lower risk of diabetes (HR 0.81, 95\% CI 0.67, 0.97). In Model 2, the $p$ value for interaction was $<0.01$ between sex and drinking status. For both sexes, among current drinkers, there was a significant decreasing trend in diabetes risk with increasing alcohol consumption in Model 2 (Table 2). Furthermore, collapsing the alcohol consumption categories among drinkers, compared with current drinkers consuming $\leq 1 \mathrm{drink} /$ week, current drinkers consuming $>1$ drink/week had a decreased risk (HR 0.86, 95\% CI 0.75, 0.99 for women; and HR 0.87, 95\% CI 0.76, 1.00 for men).

For both men and women, we found evidence for effect modification of the association of alcohol consumption and diabetes risk according to BMI $\left(p_{\text {interaction }}=0.042\right.$ for women, $p_{\text {interaction }}<$ 0.001 for men). In women, drinking $8-14$ drinks/week was associated with a decreased risk of diabetes among overweight participants (HR 0.59, 95\% CI 0.36, 0.95) (Fig. 1). Among women with obesity, drinking 2-14 drinks/week was also associated with lower risk for diabetes (HR 0.77, 95\% CI 0.60, 1.00 for 2-7 drinks/week; and HR 0.60, 95\% CI 0.37, 0.98 for 8-14 drinks/ week). In men, an inverse association was found only among obese participants who drink $>14$ drinks/week (HR $0.62,95 \%$ CI $0.43,0.89$ ).

Over the 9 year period from visit 1 to visit 4 (Fig. 2), changes in alcohol consumption status were observed. For women, $12.4 \%$ of participants changed from current to former drinkers, and $2.9 \%$ of participants changed from never to current drinkers. For men, $12.6 \%$ of participants changed from current to former drinkers, and $1.1 \%$ of participants changed from never to current drinkers. Drinking volume did not change substantially; the mean change in daily alcohol intake was -0.16 drinks $(-0.07$ drinks in women and -0.24 drinks in men). There were 1978 incident diabetes cases (1104 in women and 874 in men) which occurred during the median of 14 years of follow-up after visit 4 . For men with an alcohol intake $\geq 7$ drinks/week at baseline, a decrease in alcohol intake was associated with a higher risk of diabetes (HR 1.12, 95\% CI 1.02, 1.23 per 1 less drink/day) (Table 3). When we examined the risk of diabetes by changes in alcohol consumption status, compared with those who reported never drinking at both visits, no significant hazard ratios of incident diabetes were found (ESM Table 3).

The association between baseline drinking categories and diabetes risk were similar but somewhat weaker after excluding participants with prevalent cancer or cardiovascular disease at baseline (ESM Table 4) or when the diabetes outcome was defined using self-reported diagnosis or medication use (ESM Table 5). The somewhat weaker associations may be due to the decreased sample size and/or misclassification.

\section{Discussion}

In this community-based population, we observed a lower risk of diabetes in women who drank 8-14 drinks/week and men who drank $\geq 8$ drinks/week compared with their counterparts 
Table 1 Baseline characteristics of participants without diabetes by alcohol consumption groups, the ARIC study, 1987-1989

\begin{tabular}{|c|c|c|c|c|c|c|}
\hline & $\begin{array}{l}\text { Never drinkers } \\
(n=2802)\end{array}$ & $\begin{array}{l}\text { Former drinkers } \\
(n=2116)\end{array}$ & $\begin{array}{l}\leq 1 \mathrm{drink} / \text { week } \\
(n=2899)\end{array}$ & $\begin{array}{l}2-7 \text { drinks/week } \\
(n=2508)\end{array}$ & $\begin{array}{l}\text { 8-14 drinks/week } \\
(n=986)\end{array}$ & $\begin{array}{l}>14 \text { drinks/week } \\
(n=731)\end{array}$ \\
\hline $\begin{array}{l}\text { Alcoholic drinks per week, } \\
\text { P25-P75 }\end{array}$ & - & - & $0-0$ & $2-6$ & $9-12$ & $17-28$ \\
\hline Age (years) & $54.4(5.7)$ & $54.5(5.7)$ & $53.6(5.8)$ & $53.6(5.7)$ & $53.8(5.7)$ & $54.0(5.7)$ \\
\hline Women $(\%)$ & 76.0 & 46.8 & 64.6 & 47.7 & 33.4 & 15.5 \\
\hline \multicolumn{7}{|l|}{ Race/centre (\%) } \\
\hline White, Forsyth Co. & 27.6 & 21.0 & 26.7 & 20.7 & 22.1 & 22.3 \\
\hline White, Minneapolis & 4.7 & 18.7 & 37.8 & 42.1 & 40.6 & 34.3 \\
\hline White, Washington Co. & 26.7 & 32.2 & 26.8 & 21.6 & 24.7 & 25.7 \\
\hline Black, Forsyth Co. & 2.5 & 4.7 & 2.5 & 2.2 & 1.5 & 3.3 \\
\hline Black, Jackson & 38.5 & 23.3 & 6.1 & 13.4 & 11.1 & 14.4 \\
\hline Black $(\%)$ & 41.0 & 27.9 & 8.6 & 15.6 & 12.6 & 17.6 \\
\hline Total energy intake (kJ/day) & $6582(2856)$ & 7078 (3218) & $6638(2687)$ & $6810(2738)$ & 7079 (2649) & $8071(3233)$ \\
\hline Blood glucose level (mmol/l) & $5.4(0.5)$ & $5.5(0.5)$ & $5.4(0.5)$ & $5.5(0.5)$ & $5.6(0.5)$ & $5.6(0.6)$ \\
\hline HDL (mmol/l) & $3.0(0.9)$ & $2.7(0.8)$ & $2.9(0.9)$ & $3.0(1.0)$ & $3.1(1.1)$ & $3.0(1.0)$ \\
\hline Total cholesterol (mmol/l) & $5.6(1.1)$ & $5.5(1.1)$ & $5.5(1.1)$ & $5.5(1.0)$ & $5.6(1.0)$ & $5.6(1.1)$ \\
\hline Parental history of diabetes $(\%)$ & 25.4 & 23.3 & 21.3 & 21.1 & 20.3 & 20.8 \\
\hline Hypertension (\%) & 37.4 & 34.3 & 24.9 & 26.2 & 28.9 & 35.4 \\
\hline Coronary heart disease $(\%)$ & 2.2 & 6.9 & 3.4 & 3.6 & 5.0 & 5.1 \\
\hline BMI $\left(\mathrm{kg} / \mathrm{m}^{2}\right)$ & $28.2(5.7)$ & $27.8(5.6)$ & $26.9(4.8)$ & $26.5(4.3)$ & $26.2(4.2)$ & $26.7(4.4)$ \\
\hline Sport index & $2.3(0.7)$ & $2.4(0.8)$ & $2.5(0.8)$ & $2.6(0.8)$ & $2.7(0.8)$ & $2.5(0.8)$ \\
\hline \multicolumn{7}{|l|}{ Smoking status (\%) } \\
\hline Never & 69.9 & 28.9 & 44.8 & 32.2 & 22.0 & 12.2 \\
\hline Former & 15.5 & 41.6 & 31.4 & 39.8 & 43.5 & 41.7 \\
\hline Current & 14.6 & 29.5 & 23.9 & 28.0 & 34.5 & 46.1 \\
\hline Waist/hip ratio & $0.9(0.1)$ & $0.9(0.1)$ & $0.9(0.1)$ & $0.9(0.1)$ & $0.9(0.1)$ & $1.0(0.1)$ \\
\hline \multicolumn{7}{|l|}{ Family income (\%) } \\
\hline$<\$ 25,000$ & 50.3 & 47.7 & 25.1 & 22.7 & 21.5 & 29.4 \\
\hline$\$ 25,000-\$ 49,999$ & 36.0 & 36.1 & 42.7 & 40.2 & 36.3 & 39.0 \\
\hline$\geq \$ 50,000$ & 13.7 & 16.3 & 32.3 & 37.0 & 42.2 & 31.6 \\
\hline \multicolumn{7}{|l|}{ Education level (\%) } \\
\hline Less than high school & 28.8 & 34.3 & 13.0 & 12.6 & 15.1 & 17.6 \\
\hline $\begin{array}{l}\text { High school degree or } \\
\text { vocational school }\end{array}$ & 42.3 & 39.0 & 45.6 & 41.5 & 37.5 & 40.6 \\
\hline $\begin{array}{l}\text { At least some college or } \\
\text { professional school }\end{array}$ & 28.9 & 26.7 & 41.4 & 45.9 & 47.4 & 41.7 \\
\hline
\end{tabular}

Estimates are mean (SD) unless otherwise indicated

$\mathrm{P} 25 / \mathrm{P} 75,25$ th and 75 th percentile, respectively

who drank $\leq 1$ drink per week. There was evidence for effect modification of this association by BMI, with significant inverse associations only observed among participants who were overweight or obese. For most participants, the amount of alcohol consumed did not change substantially from visit 1 to visit 4 . A decrease in alcohol consumption among men consuming higher alcohol at baseline was associated with a higher diabetes risk.

Evidence for how alcohol consumption is related to diabetes risk may be helpful to inform the current evidence on the long-term cardiometabolic effects of alcohol. The 2015-2020
Dietary Guidelines for Americans recommend that, if alcohol is consumed, it should be moderate ( $\leq 1 \mathrm{drink} / \mathrm{day}$ for women; $\leq 2$ drinks/day for men); the guidelines do not recommend starting drinking for any reason for people who do not drink [19]. Our results suggest that any protective effect of alcohol consumption on diabetes risk is modest, may differ in men compared with women, and may differ by obesity status.

The results of our study are similar but not fully consistent with prior studies. A much earlier study on alcohol consumption and diabetes risk as part of the ARIC study found no association between moderate alcohol consumption 
Table 2 HRs of incident diabetes according to baseline alcohol consumption groups and sex

\begin{tabular}{|c|c|c|c|c|}
\hline \multirow[t]{2}{*}{ Alcohol consumption } & \multicolumn{2}{|l|}{ Women } & \multicolumn{2}{|l|}{ Men } \\
\hline & Model $1^{\mathrm{a}}$ & Model $2^{\mathrm{b}}$ & Model $1^{\mathrm{a}}$ & Model $2^{\mathrm{b}}$ \\
\hline Former drinker & $1.10(0.96,1.26)$ & $0.96(0.84,1.11)$ & $1.07(0.92,1.24)$ & $0.95(0.82,1.11)$ \\
\hline Never drinker & $1.03(0.91,1.16)$ & $0.98(0.86,1.11)$ & $0.93(0.78,1.11)$ & $0.96(0.81,1.15)$ \\
\hline$\leq 1 \mathrm{drink} /$ week & 1 (reference) & 1 (reference) & 1 (reference) & 1 (reference) \\
\hline 2-7 drinks/week & $0.82(0.71,0.95)^{*}$ & $0.88(0.77,1.02)$ & $0.94(0.81,1.08)$ & $0.93(0.81,1.08)$ \\
\hline 8-14 drinks/week & $0.71(0.56,0.91)^{*}$ & $0.75(0.58,0.96)^{*}$ & $0.89(0.75,1.07)$ & $0.84(0.70,1.00)^{*}$ \\
\hline$>14$ drinks/week & $0.86(0.58,1.27)$ & $0.91(0.61,1.34)$ & $0.92(0.77,1.11)$ & $0.81(0.67,0.97)^{*}$ \\
\hline$p$ for trend for current drinkers & 0.001 & 0.032 & 0.261 & 0.007 \\
\hline
\end{tabular}

Values are presented as HRs $(95 \% \mathrm{CI})$

${ }^{a}$ Model 1: adjusted for age, race/centre and total energy intake

${ }^{\mathrm{b}}$ Model 2: adjusted for all variables in Model 1 plus parental history of diabetes, education, household income, HDL-cholesterol, total cholesterol, baseline fasting glucose, coronary heart disease, physical activity; and time-varying BMI, smoking status (never, former, current), waist/hip ratio, and hypertension

$* p<0.05$ vs reference

and diabetes risk, while high consumption was associated with elevated diabetes risk, but only in men [12]. This prior study, however, had a relatively short follow-up period (maximum 6 years) and many fewer events compared with our analysis. The present study had a much longer followup period (maximum of 27 years) and more than three times as many incident diabetes cases, giving us greater power to detect moderate associations and to compare associations across participant subgroups. A systematic review of 20 cohort studies found U-shaped associations of alcohol with diabetes risk in both sexes, with a similar dose associated with the lowest risk at $24 \mathrm{~g}$ /day (around 1.71 drinks/day or
12 drinks/week; risk ratio $0.60,95 \%$ CI $0.52,0.69$ ) in women and $22 \mathrm{~g} /$ day (around $1.57 \mathrm{drinks} /$ day or 11 drinks/week; risk ratio $0.87,95 \%$ CI $0.76,1.00$ ) in men, respectively [7]. On the other hand, another systematic review of 38 cohort studies showed no reduction in diabetes risk in men, and found an inverse association among women with a summary hazard ratio of 0.66 , for consumption of 31-37 g/day (2.2-2.6 drinks/day) [13]. We found the lowest risk of diabetes at 1.1-2.0 drinks per day (8-14 drinks/week) in women and more than 1.1 drinks per day ( 8 drinks/week) in men. The differences in the alcohol consumption amount associated with the lowest risk among
Fig. 1 Adjusted HRs of incident diabetes by alcohol consumption group and BMI category, with numbers of participants in each category. HRs adjusted for age, race/centre, total energy intake, parental history of diabetes, education, household income, HDL-cholesterol, total cholesterol, baseline fasting glucose, coronary heart disease, physical activity; and timevarying BMI, smoking status (never, former, current), waist/hip ratio and hypertension
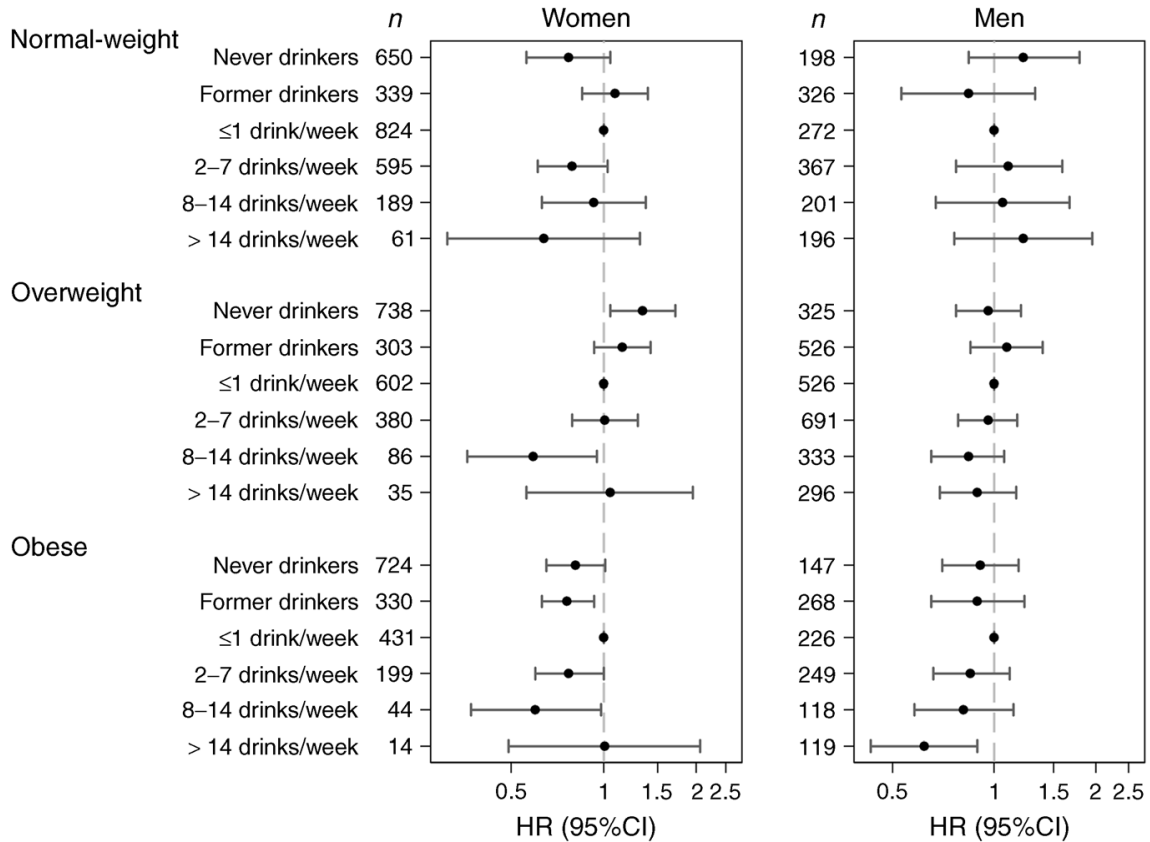

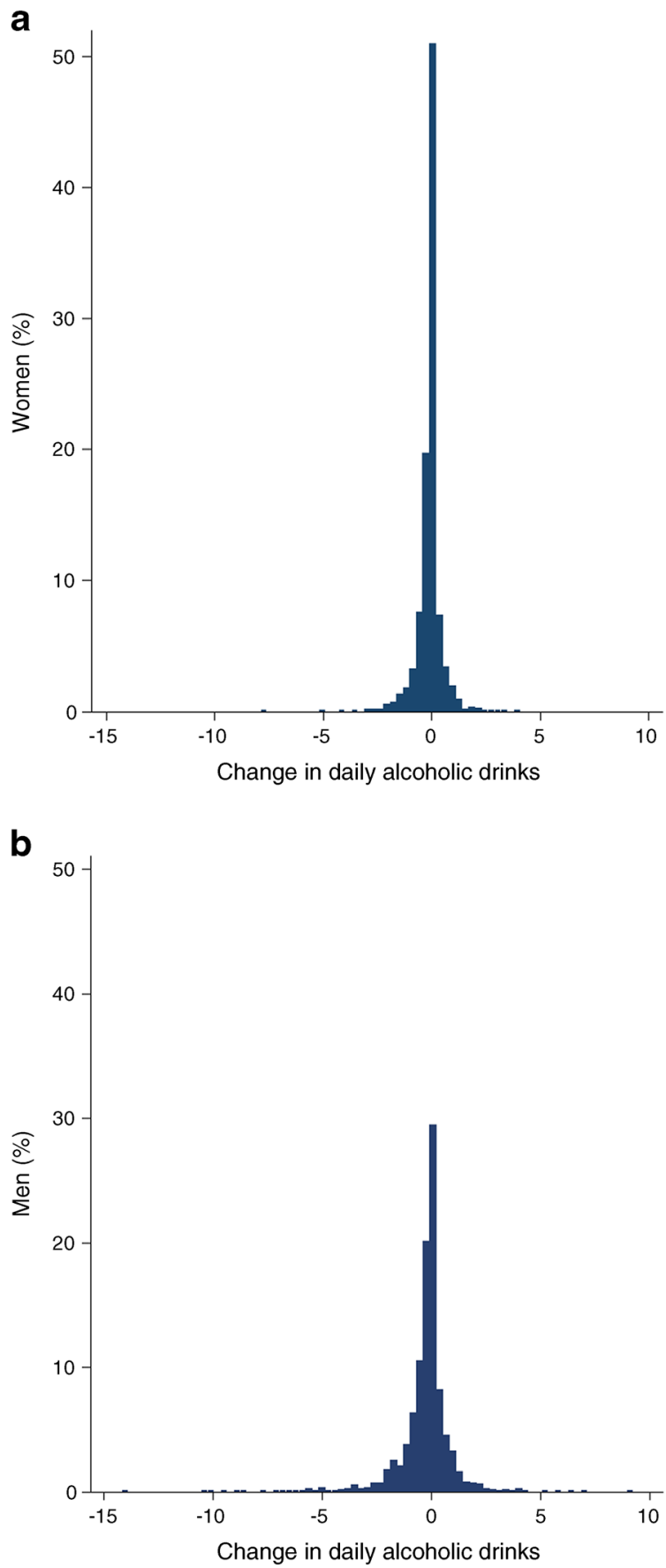

Fig. 2 Histograms of changes in number of alcoholic drinks consumed daily from visit 1 to visit 4 among women and men

the studies may be due to variability between study populations with respect to age, race, geography, duration of follow-up, and/or adjustment variables.

It is not entirely clear why BMI may be an effect modifier of the association between alcohol intake and incident diabetes. A possible explanation for this effect modification by adiposity is that obesity-induced insulin resistance is suppressed through moderate drinking [20]. Prior studies examining this question have had inconsistent findings. Our results showed decreased risk in obese and overweight women and obese men. More research is needed to further understand the mechanisms and interplay between adiposity and alcohol and their health effects.

Prior studies have speculated on the cardiometabolic effects of moderate alcohol consumption [2-4] and have demonstrated a protective effect of alcohol consumption on cardiovascular risk and cardiometabolic risk factors. There is evidence that moderate alcohol intake can increase HDLcholesterol [3], which has been postulated to partially explain the inverse association of moderate alcohol consumption with cardiovascular disease [21]. It is possible that any beneficial effects on lipids may also contribute to a decreased risk of diabetes. Some investigators have suggested that the lower risk of diabetes associated with moderate alcohol consumption may be explained by improvements in insulin sensitivity $[22,23]$. In a meta-analysis of 14 intervention studies, moderate alcohol consumption was found to be associated with lower fasting insulin concentrations and lower $\mathrm{HbA}_{1 \mathrm{c}}$ [4]. In this meta-analysis, the alcohol dosages ranged from $10 \mathrm{~g} /$ day to $75 \mathrm{~g} /$ day, and the intervention periods varied from 2 to 12 weeks [4]. Socioeconomic status is a potent risk factor for diabetes [24] and is a complex contributor to health status. Although we adjusted for major diabetes risk factors (including education, family income, CVD history, BMI and physical activity), it remains possible that the inverse associations of alcohol with cardiovascular outcomes and diabetes are the result of residual confounding $[25,26]$.

A unique aspect of our study is that we were able to investigate changes in alcohol consumption over a 9 year time window. Evidence for the association between changes in alcohol consumption and diabetes risk is limited. A prospective cohort study of men from the Health Professionals Follow-Up Study found that increases in alcohol consumption over time were associated with a lower risk of diabetes among initially light drinkers (0-4.9 g/day) [17]. Our results showed an increased diabetes risk among men with higher baseline consumption ( $\geq 7$ drinks/week) who decreased their drinking. In most participants, alcohol consumption remained stable over the 9 year period: $76 \%$ of women participants and $79 \%$ of men participants had the same alcohol consumption status (never, former or current drinkers) at baseline and at the 9 year followup visit. Our understanding of what causes lifestyle modifications such as changes in alcohol consumption in the general, middle-aged population is limited. Additional work to understand why and how adults make lifestyle changes in midlife will help inform future studies in this area.

Potential limitations of our study include the possibility of recall and/or reporting bias regarding self-reported alcohol consumption that may have resulted in misclassification of the exposure. Despite our large sample size, after stratification by sex and alcohol consumption categories, we had limited power to detect moderate associations in population 
Table 3 Adjusted HRs of incident diabetes with 1 drink daily increase or decrease of alcohol intake from visit 1 to visit 4

\begin{tabular}{|c|c|c|c|c|c|c|}
\hline \multirow[t]{2}{*}{ Change in alcohol intake } & \multicolumn{3}{|l|}{ Women } & \multicolumn{3}{|l|}{ Men } \\
\hline & Overall & $\begin{array}{l}\text { Baseline } \\
<7 \text { drinks/week }\end{array}$ & $\begin{array}{l}\text { Baseline } \geq 7 \\
\text { drinks/week }\end{array}$ & Overall & $\begin{array}{l}\text { Baseline } \\
<7 \text { drinks/week }\end{array}$ & Baseline $\geq 7$ drinks/week \\
\hline Decrease & $0.95(0.78,1.16)$ & $0.78(0.46,1.30)$ & $1.04(0.76,1.41)$ & $1.06(0.97,1.16)$ & $0.92(0.56,1.51)$ & $1.12(1.02,1.23)^{*}$ \\
\hline Increase & $0.87(0.61,1.22)$ & $1.04(0.59,1.82)$ & $0.75(0.18,3.10)$ & $1.09(0.95,1.25)$ & $1.26(0.77,2.08)$ & $1.08(0.91,1.28)$ \\
\hline No change & 1 (reference) & 1 (reference) & 1 (reference) & 1 (reference) & 1 (reference) & 1 (reference) \\
\hline
\end{tabular}

Data are presented as HRs $(95 \% \mathrm{CI})$

Adjusted for age, race/centre, total energy intake, parental history of diabetes, education, household income, HDL-cholesterol, total cholesterol, baseline fasting glucose, coronary heart disease, physical activity; and time-varying BMI, smoking status (never, former, current), waist/hip ratio and hypertension

$* p<0.05$ vs reference

subgroups, including those defined by BMI categories. High alcohol consumption was low in this population and thus, we had limited ability to examine associations in this group, especially among women (only $1.7 \%$ women were in the $>14$ drinks/week group). Because of the small numbers of individuals who changed drinking categories, the analysis of the 9 year changes were likely to be underpowered. Further, we did not assess the association between binge drinking and diabetes risk as binge drinking was only assessed at a later visit in the ARIC Study (visit 3, 1993-1995) and the prevalence of binge drinking was extremely low.

The strengths of the study include the community-based population and the long period of follow-up. We were also able to assess the effect modification by BMI of this association between alcohol consumption and diabetes risk. The wide range of rigorously measured covariates collected at baseline and later on allowed for careful adjustment of potential confounders (including time-varying covariates) and the evaluation of different adjustment models.

In conclusion, in this large, community-based cohort of black and white adults, 8-14 drinks per week for women and more than 8 drinks per week for men were associated with decreased risk of diabetes, and the associations were stronger among people with increased BMI.

Acknowledgements The authors thank the staff and participants of the ARIC study for their important contributions. The manuscript has undergone internal review and approval by the ARIC Publications Committee and ARIC Steering Committee.

Data availability The study protocol, statistical code and dataset are available from the corresponding author.

Funding The ARIC study has been funded in whole or in part with Federal funds from the National Heart, Lung, and Blood Institute, National Institutes of Health, Department of Health and Human Services, under Contract nos (HHSN268201700001I, HHSN268201700003I, HHSN268201700005I, HHSN268201700004I, HHSN268201700002I). ES was supported by NIH/NIDDK grants K24DK106414 and R01DK089174. CMR is supported by a Mentored Research Scientist Development Award from the National Institute of Diabetes and Digestive and Kidney Diseases (K01 DK107782). ML was supported by NIH/NIDDK grant R01DK089174.

Duality of interest ML was formerly a clinical site principal investigator of the Moderate Alcohol and Cardiovascular Health Trial (U10AA025286 from the National Institutes of Health) which received partial support from industry contributions to the Foundation for NIH. All other authors declare that they have no duality of interest associated with this manuscript.

Contribution statement All the authors have made substantial contributions to conception and design, acquisition of data or analysis and interpretation of data. All the authors have contributed to drafting the article or revising it critically for important intellectual content, and have given final approval for the version to be published. $\mathrm{XH}$ is the guarantor of this work.

Publisher's note Springer Nature remains neutral with regard to jurisdictional claims in published maps and institutional affiliations.

\section{References}

1. Selvin E, Parrinello CM, Sacks DB, Coresh J (2014) Trends in prevalence and control of diabetes in the United States, 19881994 and 1999-2010. Ann Intern Med 160(8):517-525. https:// doi.org/10.7326/M13-2411

2. Brien SE, Ronksley PE, Turner BJ, Mukamal KJ, Ghali WA (2011) Effect of alcohol consumption on biological markers associated with risk of coronary heart disease: systematic review and metaanalysis of interventional studies. BMJ 342:d636. https://doi.org/ 10.1136/bmj.d636

3. Rimm EB, Williams P, Fosher K, Criqui M, Stampfer MJ (1999) Moderate alcohol intake and lower risk of coronary heart disease: meta-analysis of effects on lipids and haemostatic factors. BMJ 319(7224):1523-1528. https://doi.org/10.1136/bmj.319.7224.1523

4. Schrieks IC, Heil AL, Hendriks HF, Mukamal KJ, Beulens JW (2015) The effect of alcohol consumption on insulin sensitivity and glycemic status: a systematic review and meta-analysis of intervention studies. Diabetes Care 38(4):723-732. https://doi.org/10. 2337/dc14-1556

5. Naimi TS, Stockwell T, Zhao J et al (2017) Selection biases in observational studies affect associations between 'moderate' 
alcohol consumption and mortality. Addiction 112(2):207-214. https://doi.org/10.1111/add.13451

6. Connor J, Hall W (2018) Thresholds for safer alcohol use might need lowering. Lancet 391(10129):1460-1461. https://doi.org/10. 1016/S0140-6736(18)30545-2

7. Baliunas DO, Taylor BJ, Irving $H$ et al (2009) Alcohol as a risk factor for type 2 diabetes: a systematic review and meta-analysis. Diabetes Care 32(11):2123-2132. https://doi.org/10.2337/dc090227

8. Beulens JW, van der Schouw YT, Bergmann MM et al (2012) Alcohol consumption and risk of type 2 diabetes in European men and women: influence of beverage type and body size The EPIC-InterAct study. J Intern Med 272(4):358-370. https://doi. org/10.1111/j.1365-2796.2012.02532.x

9. Djoussé L, Biggs ML, Mukamal KJ, Siscovick DS (2007) Alcohol consumption and type 2 diabetes among older adults: the Cardiovascular Health study. Obesity 15(7):1758-1765. https:// doi.org/10.1038/oby.2007.209

10. Hodge A, English D, O'Dea K, Giles G (2006) Alcohol intake, consumption pattern and beverage type, and the risk of type 2 diabetes. Diabet Med 23(6):690-697. https://doi.org/10.1111/j. 1464-5491.2006.01864.x

11. Hozawa A, Okamura T, Tanaka T et al (2010) Relation of gammaglutamyltransferase and alcohol drinking with incident diabetes: the HIPOP-OHP study. J Atheroscler Thromb 17(2):195-202. https:// doi.org/10.5551/jat.3202

12. Kao WH, Puddey IB, Boland LL, Watson RL, Brancati FL (2001) Alcohol consumption and the risk of type 2 diabetes mellitus: atherosclerosis risk in communities study. Am J Epidemiol 154(8): 748-757. https://doi.org/10.1093/aje/154.8.748

13. Knott C, Bell S, Britton A (2015) Alcohol consumption and the risk of type 2 diabetes: a systematic review and dose-response metaanalysis of more than 1.9 million individuals from 38 observational studies. Diabetes Care 38(9):1804-1812. https://doi.org/10.2337/ dc15-0710

14. Koloverou E, Panagiotakos DB, Pitsavos C et al (2015) Effects of alcohol consumption and the metabolic syndrome on 10-year incidence of diabetes: the ATTICA study. Diabetes Metab 41(2):152159. https://doi.org/10.1016/j.diabet.2014.06.003

15. Liu C, Yu Z, Li H et al (2010) Associations of alcohol consumption with diabetes mellitus and impaired fasting glycemia among middle-aged and elderly Chinese. BMC Public Health 10(1):713. https://doi.org/10.1186/1471-2458-10-713
16. Metcalf PA, Scragg RK, Jackson R (2014) Light to moderate alcohol consumption is protective for type 2 diabetes mellitus in normal weight and overweight individuals but not the obese. J Obes 2014: 634587

17. Joosten MM, Chiuve SE, Mukamal KJ, Hu FB, Hendriks HF, Rimm EB (2011) Changes in alcohol consumption and subsequent risk of type 2 diabetes in men. Diabetes 60(1):74-79. https://doi. org $/ 10.2337 / \mathrm{db} 10-1052$

18. Baecke JA, Burema J, Frijters JE (1982) A short questionnaire for the measurement of habitual physical activity in epidemiological studies. Am J Clin Nutr 36(5):936-942. https://doi.org/10.1093/ ajen/36.5.936

19. U.S. Department of Health and Human Services and U.S. Department of Agriculture (2015) Appendix 9. Alcohol. In: 2015-2020 Dietary Guidelines for Americans. 8th Edition. Available from http://health.gov/dietaryguidelines/2015/ guidelines/. Accessed 4 Apr 2018

20. Wakabayashi I (2014) Light-to-moderate alcohol drinking reduces the impact of obesity on the risk of diabetes mellitus. J Stud Alcohol Drugs 75(6):1032-1038. https://doi.org/10.15288/jsad.2014.75. 1032

21. van Tol A, Hendriks HF (2001) Moderate alcohol consumption: effects on lipids and cardiovascular disease risk. Curr Opin Lipidol 12(1):19-23

22. Furuya DT, Binsack R, Machado UF (2003) Low ethanol consumption increases insulin sensitivity in Wistar rats. Braz J Med Biol Res 36(1):125-130. https://doi.org/10.1590/S0100-879X2003000100017

23. Hendriks HF (2007) Moderate alcohol consumption and insulin sensitivity: observations and possible mechanisms. Ann Epidemiol 17(5):S40-S42. https://doi.org/10.1016/j.annepidem. 2007.01.009

24. Agardh E, Allebeck P, Hallqvist J, Moradi T, Sidorchuk A (2011) Type 2 diabetes incidence and socio-economic position: a systematic review and meta-analysis. Int J Epidemiol 40(3):804-818. https://doi.org/10.1093/ije/dyr029

25. Criqui MH, Thomas IC (2017) Alcohol consumption and cardiac disease: where are we now? J Am Coll Cardiol 69(1):25-27. https:// doi.org/10.1016/j.jacc.2016.10.049

26. Stockwell T, Zhao J, Panwar S, Roemer A, Naimi T, Chikritzhs T (2016) Do "moderate" drinkers have reduced mortality risk? A systematic review and meta-analysis of alcohol consumption and all-cause mortality. J Stud Alcohol Drugs 77(2):185-198. https:// doi.org/10.15288/jsad.2016.77.185 